\title{
Analysis of interactions between participants involved in the design of an equipment of monitoring the microclimate conditions in the automotive industry
}

\author{
Daniel-Constantin ANGHEL*, Maria Mihaela ISTRATE \\ University of Pitesti, Romania \\ *Corresponding author e-mail: daniel.anghel@upit.ro \\ Article history \\ Received 15.07.2020 \\ Accepted 20.09.2020 \\ DOI https://doi.org/10.26825/bup.ar.2020.001
}

\begin{abstract}
This paper presents a series of studies realized on a design experiment made by a multidisciplinary team from the Faculty of Mechanics and Technology of the University of Pitesti. The design experiment consisted in the development of an equipment for monitoring the microclimate conditions at a workstation from the automotive industry. The participants at this experiment had the following roles: CAD / CAM specialist, IT and electronics specialist, ergonomics specialist and a team leader. The experiment was observed and recorded in order to analyse the interactions between the participants and the effects of these interactions on the development and performance of the studied design process.
\end{abstract}

Keywords: design process, multidisciplinary team, iterations

\section{Introduction}

In the recent years, due to increasingly competitive product demands and in the context of globalization of product markets, the problem of improving the performance of the design process appear, by applying the concept of Concurrent Engineering. This concept brings a new philosophy in product development, by carrying out various activities in parallel, by multidisciplinary teams. The paper presents a series of analyses on the interaction of participants from various fields in the design of an equipment of monitoring the microclimate conditions in the automotive industry.

Having specialists from different fields has the advantage, especially for experiment, to have a large number of interactions to explain the specific interventions required during the development of the experiment, and solving conflicts between professions.

\section{Risks associated to the concept of concurrent engineering in terms of interaction between participants}

One way to reduce the durations is to perform as many activities is possible at the same time. However, precisely due to the fact that certain activities are performed in parallel, by multidisciplinary teams, in case of need to repeat one of the activities, the risk of repetition is higher than in the case of the classic approach, where a smaller number of activities was repeated.

Repeating activities requires iterations in the design process, iterations that lead to longer durations and increased design costs. Osborne [OSBO 92] says that iterations means between $13 \%-70 \%$ of the total time consumed with the design activity at Intel. 
Browning [BROW 98] defines iteration as a repetition of activities to improve a partial solution. Thus, optimizing the interaction between participants leads to a decrease in the number of iterations required to improve a solution [ANGH 04].

Stewart [STEW 92] shows that 75\% of design issues are caused by human errors. Kaminetzky [KAMY 91] classifies human errors into errors of interpretation, errors of performance, and errors of intent. It identifies the following types of human errors, table 1.

Table 1. Typology of human errors

\begin{tabular}{|l|l|}
\hline Types of human errors & Causes of errors \\
\hline Method error & Choosing an incorrect method for analysis \\
\hline Inadequate technique & $\begin{array}{l}\text { The method does not have the required } \\
\text { accuracy }\end{array}$ \\
\hline Activity not performed & Failure to perform a specific design activity \\
\hline Coding error & Improper use of a standard \\
\hline Signal loss & An incorrectly reproduced scheme \\
\hline Noise in the signal & Phone conversation in a noisy room \\
\hline Decoding error & $\begin{array}{l}\text { The user does not understand the notations in } \\
\text { the standard or on a drawing }\end{array}$ \\
\hline
\end{tabular}

\section{Type of interaction between participants of the design experiment}

This experiment was made at University of Pitești, at the Faculty of Mechanics and Technology in a Design and Development Product Laboratory.

The subject of this experiment was to realise an equipment for monitoring the microclimate conditions at a workstation, and the main objective was to observe the experiment in order to identify all the type of interactions between the participants. At this experiment four participants were involved.

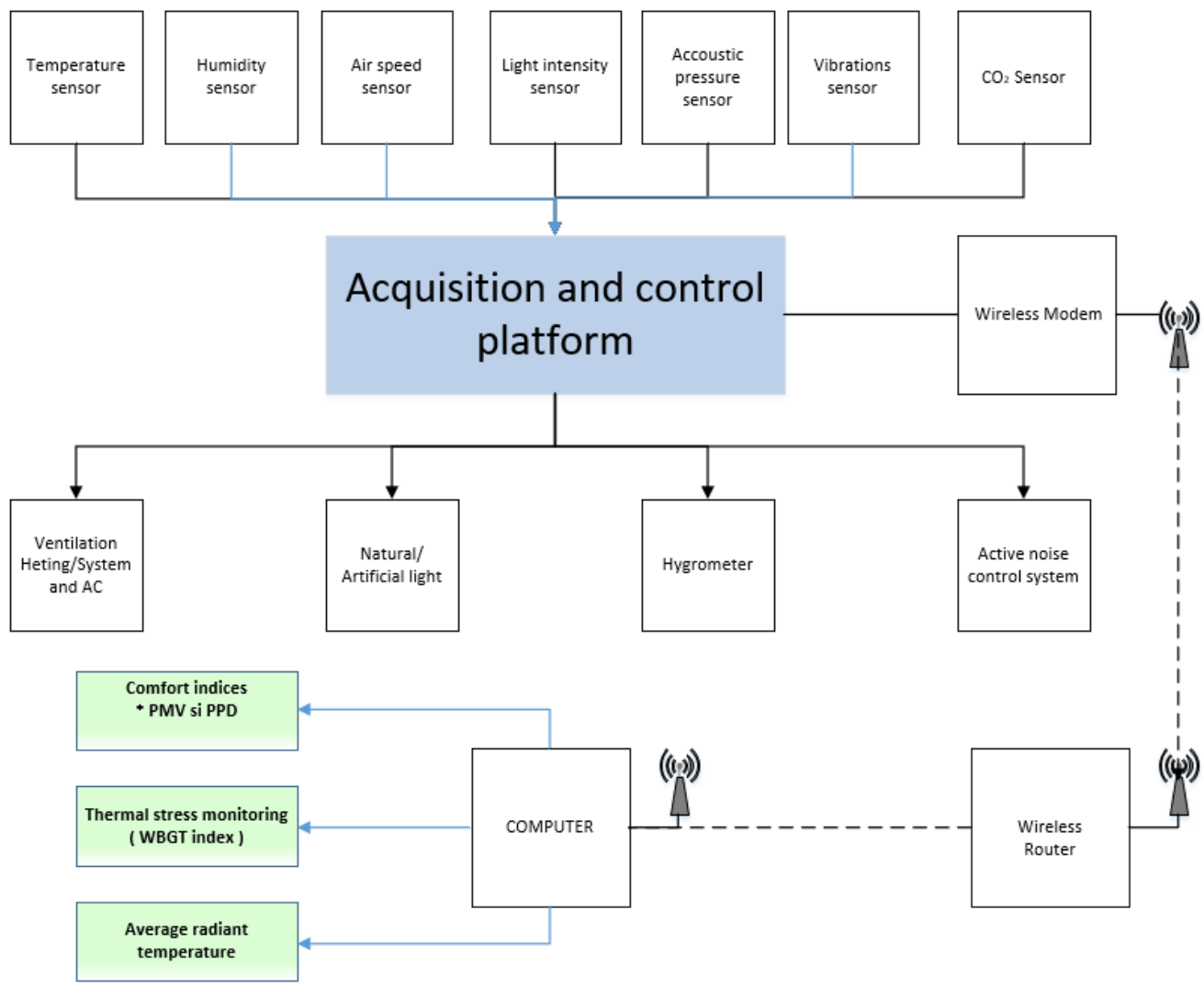

Figure 1. The equipment of monitoring the microclimate conditions

The design process for this experiment was divided into 14 design task: 
1 Establishing the data necessary to monitoring a workstation;

2 Establishing the data necessary for the design of the equipment;

3 Choice of platform and the sensors;

4 Acquisition of components;

5 Establishing the sensor reading mode;

6 Equipment body design;

7 Realization of programs for 3D printing of the equipment body;

8 Printing the body of the equipment;

9 Assembling the equipment;

10 Realization of sensor reading programs;

11 Platform testing and calibration;

12 Realization of the acquisition and processing programs of the obtained data;

13 Establishing the way of displaying alerts and communicating reports;

14 Description of operation and technical prescriptions.

The participants at this experiment had the following roles: CAD / CAM specialist (CS), IT and electronics specialist (IS), ergonomics specialist (ES) and a team leader (TL).

The possibilities of interaction between the participants in the experiment are:

a. TL $\rightarrow$ CS, IS and ES The team leader presents to the team members the objectives and the main stages of the project;

b. TL $\rightarrow$ CS and IS $\rightarrow$ ES Where the ES is required for its status as an expert in ergonomics to make a diagnosis, validating or invalidating certain solutions;

c. TL $\rightarrow$ CS and ES $\rightarrow$ IS Where the IS is required for its status as an expert in IT and electronics to make a diagnosis, validating or invalidating certain solutions;

d. TL $\rightarrow$ IS and ES $\rightarrow$ CS In this case, the IS intervention is shifted upstream, being able to open the solution space, being able to propose a new technology for replacing a certain sensor;

e. ES $\rightarrow$ CS, IS and TL It is the case when the ES takes the initiative to evaluate a solution and communicate the results directly to the TL and / or to the other specialists;

f. CS $\rightarrow$ ES, IS and TL It is the case when the CS takes the initiative to propose directly an idea or a solution to the TL and / or to the other specialists;

g. TL $\rightarrow$ CS or IS or ES TL sends directives to one of the specialists;

h. CS or IS or ES $\rightarrow$ TL It is the case when one of the specialists launches an idea to TL;

i. $\quad$ CS $\rightarrow$ IS CAD / CAM specialist presents a solution to IT and electronics specialist;

j. $\quad$ CS $\rightarrow$ ES CAD / CAM specialist presents a solution to ergonomics specialist;

k. ES $\rightarrow$ IS ergonomics specialist presents a solution to IT and electronics specialist;

1. ES $\rightarrow$ CS ergonomics specialist presents a solution to CAD / CAM specialist;

m. IS $\rightarrow$ CS IT and electronics specialist presents a solution to CAD / CAM specialist;

n. IS $\rightarrow$ ES IT and electronics specialist presents a solution to ergonomics specialist. 


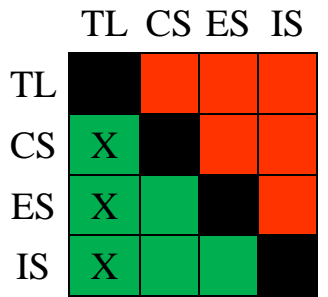

a.
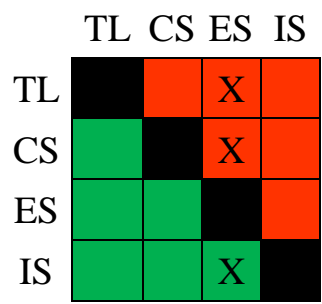

e.
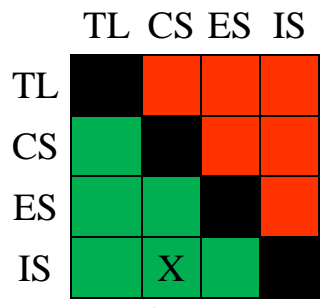

i.

TL CS ES IS

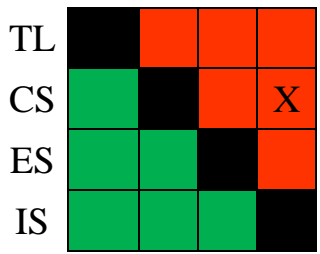

$\mathrm{m}$.

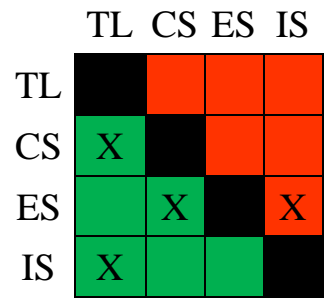

b.
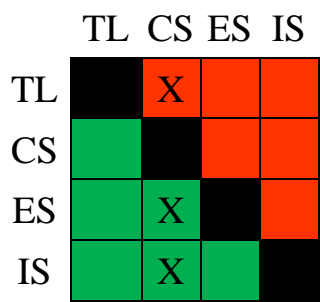

f.

TL CS ES IS

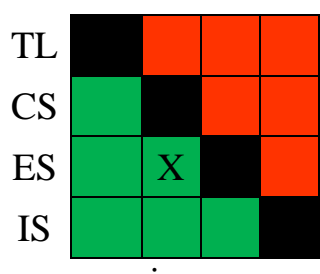

j.

TL CS ES IS

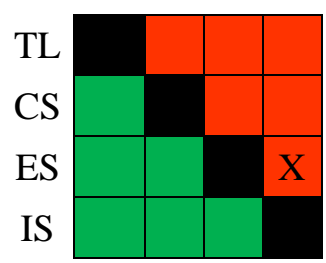

n.
TL CS ES IS

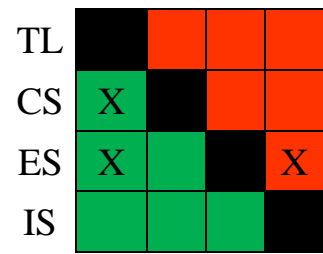

c.
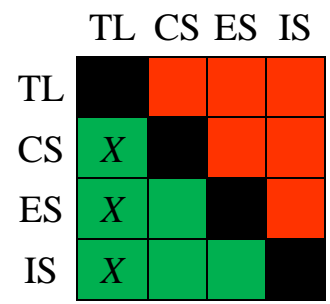

g.
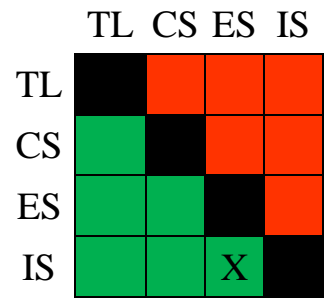

$\mathrm{k}$.

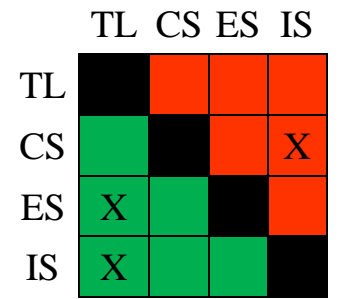

d.

TL CS ES IS

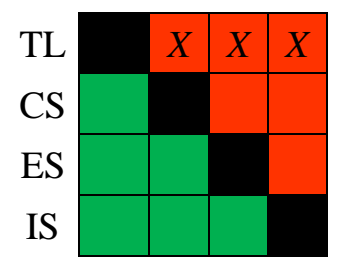

h.

TL CS ES IS

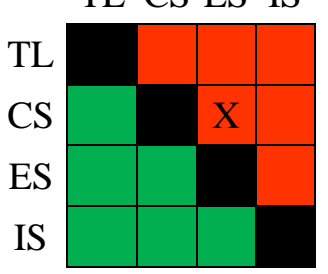

1.

Figure 2. Matrix representation of the interactions between the participants of the design experiment

In figure 2 we observe that on the matrix has two different zones: zone below the main diagonal of the matrix, la zone green, represent le flux direct of information and the zone above the main diagonal of the matrix, la zone rouge, represent le flux indirect of information, that is, iterations.

Many of the interactions between the participants on the design experiment have an iterative or even purely iterative character, which shows that the process is a "disturbed" one.

The experiment was performed during a working week, requiring a number of 1250 minutes.

In the figure 3 we present "a negotiation" between participants, type "f". In this case, CAD / CAM specialist (CS) make a proposition on the architecture of the housing of the equipment in order to establish the pertinent place for the BMP280 sensor. In this case, an iteration is made, because the team leader with IS and ES must analyze and validate this new proposition.

The analyze must taking account the following criteria:

- The functionality of the sensor must be assured;

- The sensor must be protected against blows, water, sharp objects etc.;

- The architecture of the equipment housing must be impacted as minimum as possible;

- The BMP280 sensor must be easy install or uninstall on the equipment; 


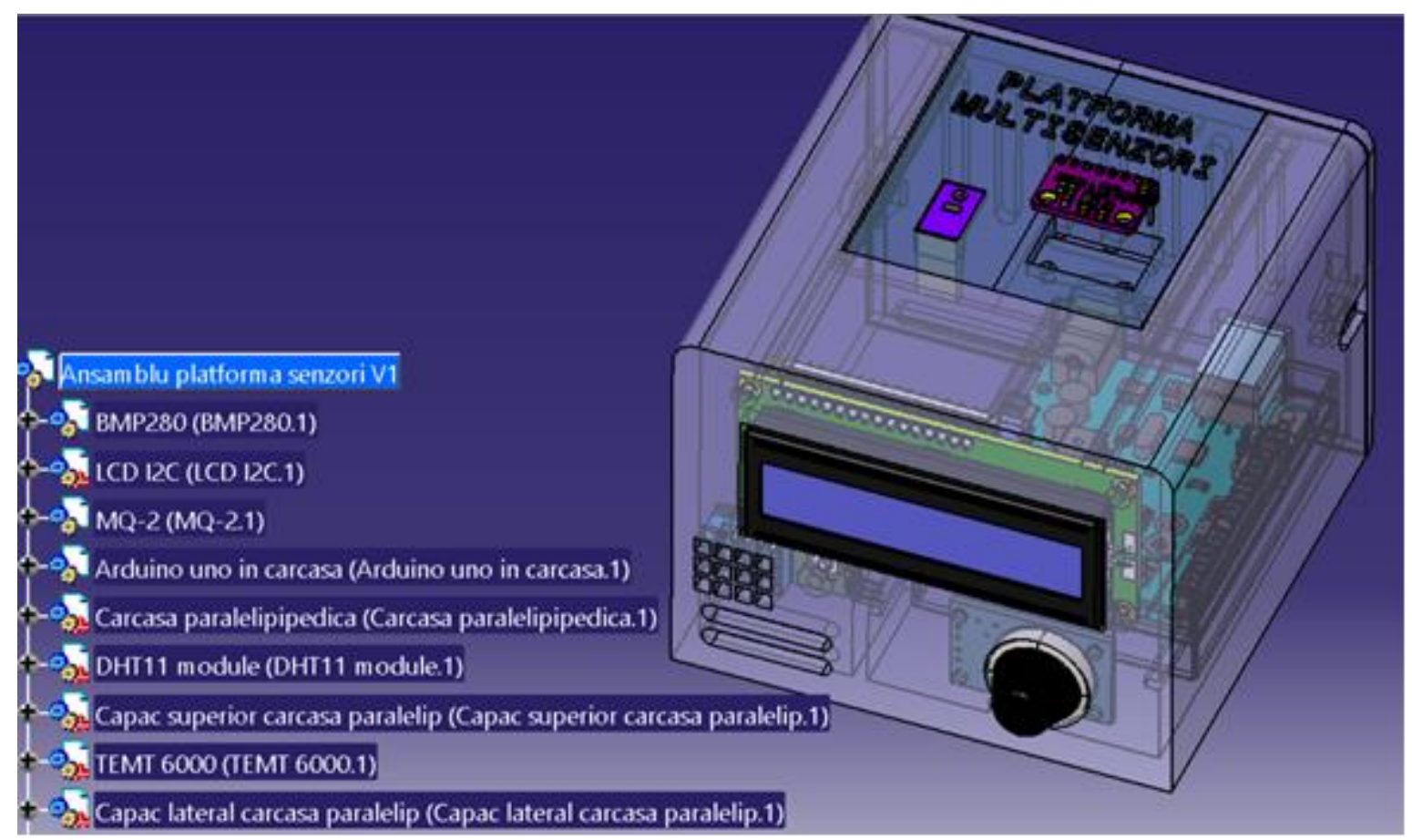

Figure 3. Negotiation for the "appropriate" emplacement of BMP280 sensor

On the figure 4. we observe de durations of each design task. The task with the biggest duration is the task number 6, Equipment body design.

During this task we identified for type of interaction between participants:

- Type "a", with a ratio of $10 \%$ of total duration of the task;

- Type "f", with a ratio of $40 \%$ of total duration of the task;

- Type "h", with a ratio of $30 \%$ of total duration of the task. This kind of interaction generate an iteration process, necessary to solve the problems generated by the particularities of each profession;

- Type "j", with a ratio of $20 \%$.

\begin{tabular}{|c|l|c|}
\hline \multicolumn{1}{|c|}{ Design task } & Task duration \\
\hline \multicolumn{2}{|l|}{ (min) } \\
\hline 1 & Establishing the data necessary to monitoring a workstation; & 40 \\
\hline 2 & Establishing the data necessary for the design of the equipment; & 30 \\
\hline 3 & Choice of platform and the sensors; & 80 \\
\hline 4 & Acquisition of components; & 100 \\
\hline 5 & Establishing the sensor reading mode; & 70 \\
\hline 6 & Equipment body design; & 240 \\
\hline 7 & Realization of programs for 3D printing of the equipment body; & 30 \\
\hline 8 & Printing the body of the equipment; & 210 \\
\hline 9 & Assembling the equipment; & 90 \\
\hline 10 & Realization of sensor reading programs; & 70 \\
\hline 11 & Platform testing and calibration; & 30 \\
\hline 12 & Realization of the acquisition and processing programs; & 60 \\
\hline 13 & Establishing the way of displaying alerts and communicating reports; & 80 \\
\hline 14 & Description of operation and technical prescriptions. & 120 \\
\hline
\end{tabular}

Figure 4. The durations of the tasks of the design experiment 
On the figure 5 the ratios of each type of intervention on the tasks of the design experiment is presented. We can observe that the biggest number of type of interventions was made on the task 14, Description of operation and technical prescription. For realizing this task, a lot of iterative loops was necessary, in order to negotiate the propositions of each participant and to establish the appropriate form of the document.

\begin{tabular}{|c|c|c|c|c|c|c|c|c|c|c|c|c|c|c|c|}
\hline & & \multicolumn{14}{|c|}{ Type of interactions \% } \\
\hline \multicolumn{2}{|r|}{ Design task } & $a$ & b & C & d & e & $f$ & $\mathrm{~g}$ & $\mathrm{~h}$ & $\mathrm{i}$ & j & $\mathrm{k}$ & I & $\mathrm{m}$ & $\mathrm{n}$ \\
\hline 1 & Establishing the data necessary to monitoring a workstation; & 0,8 & & & & & & & & 0,1 & 0,1 & & & & \\
\hline 2 & Establishing the data necessary for the design of the equipment; & 0,2 & & & & 0,4 & 0,3 & 0,1 & & & & & & & \\
\hline 3 & Choice of platform and the sensors; & 0,1 & & & & 0,2 & & & & & & & & 0,7 & \\
\hline 4 & Acquisition of components; & 0,2 & 0,3 & 0,2 & 0,2 & & & & & & & & & 0,1 & \\
\hline 5 & Establishing the sensor reading mode; & & & & & & & & & & & & & 0,4 & 0,6 \\
\hline 6 & Equipment body design; & 0,1 & & & & & 0,4 & & 0,3 & & 0,2 & & & & \\
\hline 7 & Realization of programs for $3 D$ printing of the equipment body; & & & & & & 0,8 & & & & 0,2 & & & & \\
\hline 8 & Printing the body of the equipment; & & & & & & 1,0 & & & & & & & & \\
\hline 9 & Assembling the equipment; & & & & & & & & & & & & & & \\
\hline 10 & Realization of sensor reading programs; & & & 0,1 & 0,2 & & & & & 0,1 & & 0,2 & 0,1 & 0,2 & 0,1 \\
\hline 11 & Platform testing and calibration; & & 0,1 & & 0,1 & & & & 0,2 & 0,1 & & & 0,2 & 0,2 & 0,1 \\
\hline 12 & Realization of the acquisition and processing programs; & 0,1 & & & & & & & & & & & & 0,4 & 0,5 \\
\hline 13 & Establishing the way of displaying alerts and communicating reports; & 0,2 & & & & & & & & & & & & 0,3 & 0,6 \\
\hline 14 & Description of operation and technical prescriptions. & & 0,1 & 0,1 & 0,2 & 0,1 & & 0,1 & & & 0,1 & 0,1 & & 0,2 & \\
\hline
\end{tabular}

Fig. 5. Ratio of each type of intervention on the tasks of the design experiment

\section{Conclusions}

Although the designed product is a product in the field of ergonomics, the analysis found that the most involved specialist was CAD / CAM specialist (CS).

The role of the ergonomics specialist (ES) was rather that of a consultant, especially for the IT and electronics specialist (IS).

In order to improve performances of the design process, an improvement of the interactions between the participants can be achieved by involving the team leader (TL) in mediating the interactions between the participants and by stimulating them to participate in as many design tasks as possible.

\section{References}

ANGHEL D.C., Boudouh T., Garro O., Ungureanu I.,2004, Minimizing iteration impacts in the design process, 10th International Conference on Concurrent Enterprising, Escuela Superior de Ingenieros, Seville, Spain, 14-16 June 2004.

BROWNING, T., R.,1998, Use of Dependency Structure Matrices for Product Development Cycle Time Reduction, Proceedings of the Fifth ISPE International Conference on Concurrent Engineering: Research and Applications, Tokyo, Japan, July 15-17, 1998.

KAMINETZKY, D.,1991, "Design and Construction Failures: Lessons from Forensic Investigations", McGraw Hill, New York, 1991.

OSBORNE, S.M.,1992. Product development cycle time characterization through modeling of process iteration. Master's Thesis, Massachusetts Institute of Technology.

STEWART, M.G., 1992, "Simulation of human error in reinforced concrete design", Research in Engineering Design 4(1), 1992, pp.51-60. 